\title{
EXCITATION OF COUPLED STELLAR MOTIONS IN THE GALACTIC DISK BY ORBITING SATELLITES
}

\author{
E. D’Onghia ${ }^{1,6}$, P. Madau ${ }^{2}$, C. Vera-Ciro ${ }^{3}$, A. Quillen ${ }^{4}$, and L. Hernquist ${ }^{5}$ \\ ${ }^{1}$ Department of Astronomy, University of Wisconsin, 2535 Sterling Hall, 475 N. Charter Street, Madison, WI 53076, USA; edonghia@astro.wisc.edu \\ ${ }^{2}$ University of California Santa Cruz, 1156 High Street, Santa Cruz, CA 95064, USA \\ ${ }^{3}$ Departamento de Ciencias Basicas, Universidad de Medellin, Cra 87 N30-65, Medellin, Colombia \\ ${ }^{4}$ Department of Physics and Astronomy, University of Rochester, Rochester, NY 14627, USA \\ ${ }^{5}$ Harvard-Smithsonian Center for Astrophysics, 60 Garden Street, MS-51, USA \\ Received 2015 September 21; accepted 2016 March 9; published 2016 May 13
}

\begin{abstract}
We use a set of high-resolution N-body simulations of the Galactic disk to study its interactions with the population of cosmologically predicted satellites. One simulation illustrates that multiple passages of massive satellites with different velocities through the disk generate a wobble, which has the appearance of rings in face-on projections of the stellar disk. They also produce flares in the outer disk parts and gradually heat the disk through bending waves. A different numerical experiment shows that an individual satellite as massive as the Sagittarius dwarf galaxy passing through the disk will drive coupled horizontal and vertical oscillations of stars in underdense regions with small associated heating. This experiment shows that vertical excursions of stars in these low-density regions can exceed $1 \mathrm{kpc}$ in the Solar neighborhood, resembling the recently locally detected coherent vertical oscillations. They can also induce non-zero vertical streaming motions as large as $10-20 \mathrm{~km} \mathrm{~s}^{-1}$, which is consistent with recent observations in the Galactic disk. This phenomenon appears as a local ring with modest associated disk heating.
\end{abstract}

Key words: galaxies: kinematics and dynamics - Galaxy: disk - Galaxy: evolution - stars: kinematics and dynamics

Supporting material: animation

\section{INTRODUCTION}

The nature of the vertical bulk motion of stars recently discovered in the solar neighborhood remains uncertain. These motions assume the form of vertical oscillations of the stellar disk as found by the RAdial Velocity Experiment and in the Large Area Multi-Object Spectroscopic Telescope radial velocity survey (Widrow et al. 2012; Carlin et al. 2013; Williams et al. 2013). In addition, Widrow et al. (2012) and Yanny \& Gardner (2013) find evidence for wave-like northsouth asymmetries in the number counts of stars in the solar neighborhood. The origin of these non-zero average vertical velocities is controversial and different studies advocate for a variety of formation scenarios, from being caused by the transient nature of the spiral structure (Debattista 2014), to the presence of the bar (Monari et al. 2014, 2015), to external perturbations, such as the Sagittarius dwarf galaxy (Gómez et al. 2012, 2013, 2016; Widrow et al. 2014; de la Vega et al. 2015).

In a hierarchical universe in which disks are believed to form and evolve, galaxies are surrounded by a population of orbiting luminous and dark satellites, with $\sim 10 \%-15 \%$ of them expected to pass through the primary disks (D'Onghia et al. 2010a) and perturb them. In particular, the interaction of a stellar disk with visible dwarf satellite galaxies has been suggested as a way to excite structures in galactic disks, such as rings, warps, spiral structures, while also providing mechanisms to dynamically heat stellar disks (Quinn et al. 1993; Walker et al. 1996; Younger et al. 2008; Chakrabarti \& Blitz 2009; Kazantzidis et al. 2009; Purcell et al. 2011; Struck et al. 2011; Bird et al. 2012; Gómez et al. 2012).

\footnotetext{
${ }^{6}$ Alfred P. Sloan Fellow.
}

An early study by Toth \& Ostriker (1992) computed the energy deposited locally into a disk and halo through a merger with a satellite galaxy on a nearly circular orbit, decaying by dynamical friction. However, self-gravitating disks support collective modes, such as density waves propagating across and perpendicular to the plane, which are neglected in the impulse approximation or dynamical friction formalism but are naturally described in N-body experiments. Later numerical simulations indicated that disks could be strongly heated by the accretion of massive satellites and included other effects, previously ignored, such as the tidal disruption of the satellite as it approaches the host galaxy and the tilting of the disk plane due to the gravitational torque from the satellite. However, some of the early calculations suffered from numerical relaxation and noise (Sellwood 2013). Low mass and spatial resolution make a disk unable to support collective modes, casting doubt on the outcome of the numerical experiments.

The vertical structure and thickening of galaxy disks due to the accretion of small satellites was explored by Sellwood et al. (1998), who suggested that this process can excite large-scale bending waves in a disk. The associated vertical heating involves the dissipation of bending waves at resonances with stellar orbits in the disk. More recent numerical experiments found that under realistic circumstances, the vertical heating of disks scales with the satellite to disk mass ratio as $\sim\left(M_{\text {sat }} / M_{\text {disk }}\right)^{2}$ (Hopkins et al. 2008). Therefore, it is expected that disk heating is caused primarily by the high-mass end of the satellite distribution predicted in $\Lambda \mathrm{CDM}$ cosmological simulations and is reduced in the solar neighborhood (Moetazedian \& Just 2015) relative to early estimates (Hopkins et al. 2009).

One assumption often made in studies of disk dynamics is that the vertical heating of a disk is largely unaffected by the 
Table 1

Galaxy Structural Parameters

\begin{tabular}{lccc}
\hline \hline Parameters & Experiment 1 & Experiment 2 & Experiment 3 \\
\hline$a(\mathrm{kpc})^{\mathrm{a}}$ & 29.6 & 29.6 & 29.6 \\
$R^{\mathrm{b}}(\mathrm{kpc})^{\mathrm{b}}$ & 4.0 & 3.13 & 3.13 \\
$M_{\mathrm{DM}}\left(M_{\odot}\right)^{\mathrm{c}}$ & $9.5 \times 10^{10}$ & $9.5 \times 10^{10}$ & $9.5 \times 10^{10}$ \\
$M_{\text {disk }}\left(M_{\odot}\right)^{\mathrm{d}}$ & $1.8 \times 10^{10}$ & $1.8 \times 10^{10}$ & $1.8 \times 10^{10}$ \\
$z_{\text {disk }}(\mathrm{kpc})^{\mathrm{e}}$ & 0.4 & 0.313 & 0.313 \\
$N_{\text {sat }}$ & 1 & 1000 & $\sim 100$ \\
$M_{\text {sat }}\left(M_{\odot}\right)^{\mathrm{g}}$ & $2 \times 10^{10}$ & $9.5 \times 10^{6}$ & $\left({ }^{\mathrm{h}}\right)$ \\
\hline
\end{tabular}

Notes.

${ }^{a}$ Halo scale length.

b Stellar disk length.

${ }^{\mathrm{c}}$ Dark matter halo total mass.

d Stellar disk total mass.

e Disk scale heigth.

${ }^{\mathrm{f}}$ Number of satellites.

g Satellite mass.

${ }^{\mathrm{h}}$ The satellite mass distribution follows a power law: $d N / d M \propto M^{-1.9}$.

energy deposited in the motion in the plane, and that the vertical and horizontal motions are not coupled. Based on this assumption, the vertical heating of stellar disks has always been considered a relatively simple problem, since the stars are not expected to be influenced in their vertical oscillations by the energy deposited into their radial motions.

In this paper, we study the effects of the passage of satellites through stellar disks when self-gravity is included, and examine the consequences for disk-thickening and the kinematic signatures of stars in the solar neighborhood. We show that when at least one massive satellite impacts the disk, the horizontal and vertical motions are locally coupled, which has implications for the vertical structure and the heating of stellar disks.

\section{NUMERICAL METHODS}

To investigate the gravitational response of a disk to the impact of dark satellites, we have performed a total of three $\mathrm{N}$-body experiments in which a live disk of $10^{8}$ star particles was embedded in a spherical dark halo with the introduction of one satellite dark galaxy as massive as Sagittarius, and in subsequent experiments with many substructures as extracted from cosmological simulations.

The dark-matter distribution of the host galaxy is modeled with a Hernquist profile (Hernquist 1990) with a total halo mass of $9.5 \times 10^{11} M_{\odot}$ and a scale length of $29.6 \mathrm{kpc}$ (see Table 1).

The stellar disk in the initial conditions of all experiments is described by an exponential surface density profile: $\Sigma_{*}(R)=M_{*} /\left(2 \pi R_{d}^{2}\right) \exp ^{\left(-R / R_{d}\right)}$, with $R_{d}$ being the scale length. The vertical mass distribution of the stars in the disk is specified by the profile of an isothermal sheet with a radially constant scale height $z_{\text {disk }}$. The three-dimensioal (3D) stellar density in the disk is then

$$
\begin{aligned}
\rho_{*}(R, z) & =\Sigma(R) \zeta(z) \\
& =\frac{M_{*}}{4 \pi z_{\text {disk }} R_{d}^{2}} \operatorname{sech}^{2}\left(\frac{z-z_{0}}{z_{\text {disk }}}\right) \exp \left(-\frac{R}{R_{d}}\right) .
\end{aligned}
$$

Here, $z_{0}$ is the first moment of the $\mathrm{z}$ component of stars, $z_{\text {disk }}$ is treated as a free parameter that is set by the vertical velocity dispersion of the stars in the disk, and the velocity distribution of the stars is chosen such that this scale height is selfconsistently maintained in the full 3D potential of the galaxy (Hernquist 1993; Springel et al. 2005). A value of $z_{\text {disk }}=0.1$ $R_{d}$ is adopted. The parameters describing the total halo mass and the stellar disk are summarized in Table 1. The details of the rotation curve of each galaxy component and the behavior of the Toomre parameter $Q$ as a function of radius are reported in Figure 1 of D'Onghia et al. (2013) and Vera-Ciro et al. (2014). The disk mass fraction within two scale-lengths is $30 \%$. With this choice of structural parameters, the outcome is a multi-armed spiral disk galaxy with no stellar bar (D’Onghia 2015).

Unlike in D'Onghia et al. (2013), where the disk was perturbed by co-rotating overdense regions with masses similar to giant molecular clouds, here instead we have explored the dynamical response of the disk to interactions with one or many substructures.

First Experiment. We added to the stellar disk only one live substructure as massive as the Sagittarius dwarf galaxy, with a mass of $2 \times 10^{10} M_{\odot}$ and hitting the disk at roughly at $2.2 \mathrm{kpc}$ from the disk center. The disk was surrounded by a live darkmatter halo in this case. The satellite was placed on an eccentric orbit $(e=0.7)$ at an initial distance of $20 \mathrm{kpc}$ below the plane. It completed one orbit around the disk in approximately 1.7 Gyrs.

Second Experiment. We added 1000 substructures with a mass of $10^{7} M_{\odot}$, hitting the disk from random orbits, to the live disk embedded in a static potential for the halo.

Third Experiment. In this experiment, the same galaxy model was evolved but with the introduction of $\approx 100$ live dark satellites isotropically distributed. In this set-up, we have at least two satellites with masses larger than $10^{9}$ and several satellites with masses larger than $2 \times 10^{8} M_{\odot}$ which pass through the disk.

The $\approx 100$ satellite halos have masses in the range $M_{\text {sat }}=2 \times 10^{8}-2 \times 10^{10} M_{\odot}$ orbiting around a stellar disk with a mass of $1.9 \times 10^{10} M_{\odot}$ embedded in a static dark halo. The mass distribution of the substructures is characterized by a power law of $d N / d M \propto M^{-1.9}$, as expected from cosmological simulations (Diemand et al. 2008; Springel et al. 2008). The cumulative mass of all of the subhalos is $10 \%$ of the total mass of the galaxy halo.

Each substructure consists of a live dark-matter halo modeled assuming a Hernquist profile, with a scale-radius, $a$, related to the Navarro-Frenk-White profile through the relation (Springel et al. 2005) $a=r_{s} \sqrt{2[\ln (1+c)-c /(1+c)]}$, where $c$ is the subhalo concentration as predicted in cosmological simulations. The number of particles ranges from $10^{3}$ to a maximum of $5 \times 10^{5}$ particles for the most massive substructure.

\section{RESULTS}

\subsection{Dynamical Response of the Disk to Satellite Perturbations}

We first verified that low-mass disks run with sufficiently large numbers of particles do not develop spiral structures in the absence of any perturbing influence (D'Onghia et al. 2013).

Figure 1 shows the outcome of the first experiment where the disk is displayed face-on after being hit by a massive darkmatter substructure. We note that the disk initially develops an 

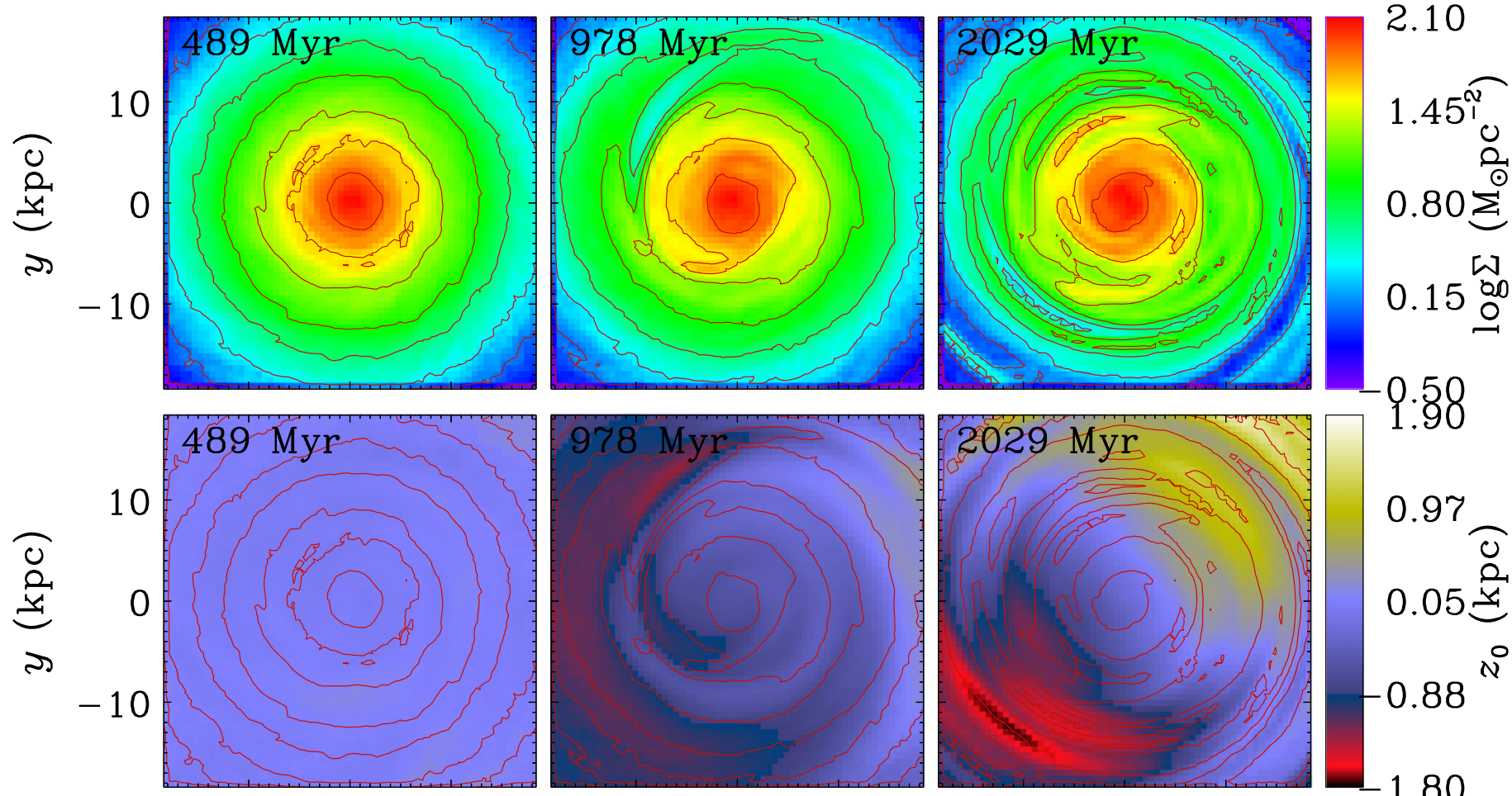

1:50
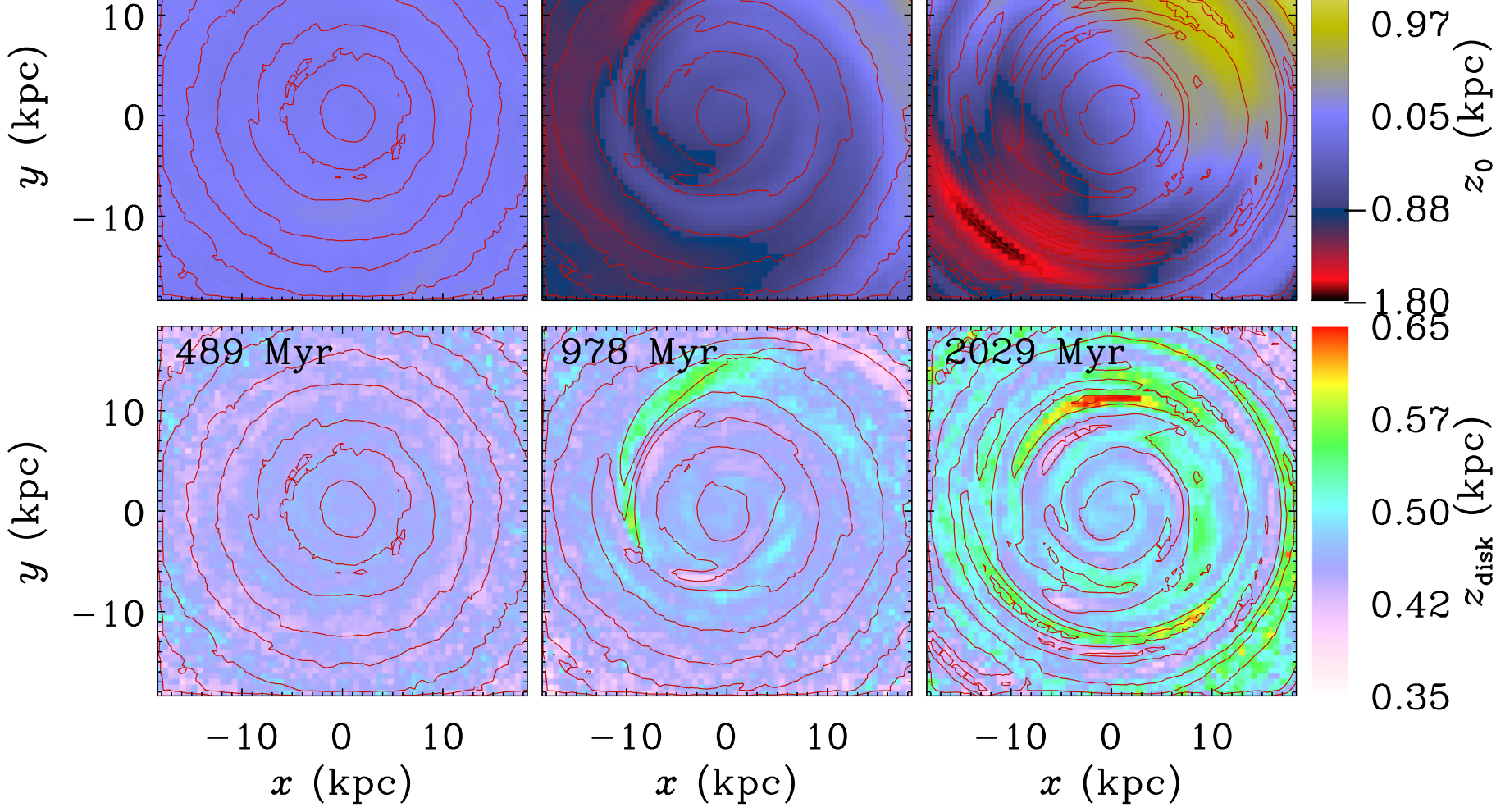

Figure 1. Time evolution of the stellar disk seen face-on perturbed by one satellite of mass $2 \times 10^{10} M_{\odot}$. The surface mass density values are displayed (top panels). Time evolution of the same disk face-on with the first moment of the vertical component of the stars shown (middle panels) and enhancements of the thickness of the disk displayed with their values (bottom panels).

(An animation of this figure is available.)

open, two-armed pattern which propagates outwards to form a ring. The satellite is massive enough that its impact causes the disk to respond immediately after the intruder passes, with the stars around the collision site moving inward and then expanding outward. As a result of the contraction and expansion, there is a wave of enhanced density that moves and forms a pattern similar to a ring (top panels of Figure 1). Indeed, this process is thought to be responsible for rings in galaxies through off-center collisions, but not the structures that resemble the patterns we see in nearby spiral galaxies (Lynds \& Toomre 1976; Hernquist \& Weil 1993; D’Onghia et al. 2008; Mapelli \& Mayer 2012). Similar features are produced in the simulation following several satellites passing through the disk.
To better understand the forming pattern in our simulations, we consider a heuristic model. We assume a displacement of the disk in $z$ with a function proportional to $e^{i m \phi}$. Then, the ring pattern rotates with a pattern speed:

$$
\Omega_{p}=\Omega-\frac{\nu_{z}}{m}
$$

where $\nu_{z}$ is the halo vertical oscillation frequency (see the derivation below for the Hernquist model) and $\Omega(R)$ is the angular rotation rate. For $m=1$, we expect $\nu_{z}(R)>\Omega(R)$ with a retrograde ring pattern.

Note that the pattern is expected to move with angular rotation rate $\left(\Omega-\nu_{z}\right)$, with its derivative with respect to the radius being the sheer rate for the vertical disturbance. For an 

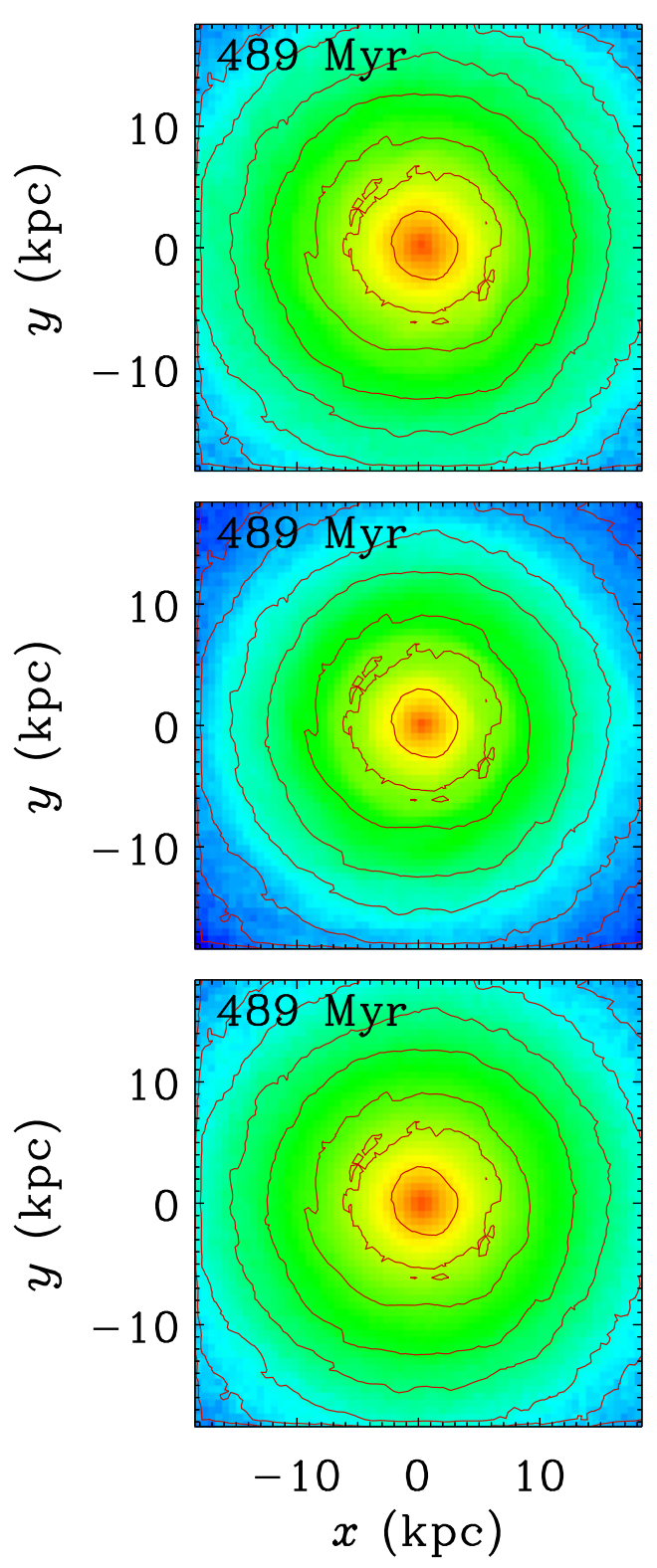

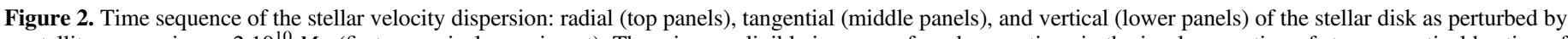

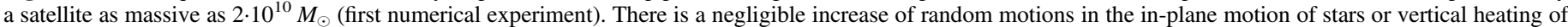
the disk, even after more than $2.5 \mathrm{Gyr}$.

initial perturbation at a given azimuthal angle with size $\Delta R$, after a time $\Delta t$, the perturbation will be wrapped in $2 \pi$ across a radial distance:

$$
\Delta t \sim \frac{2 \pi}{\left(\nu_{z}-\Omega\right) \Delta R} \approx R \frac{T}{\Delta T}
$$

where $T$ is the orbital period for stars. At $R=2.2 \mathrm{kpc}$, $T=0.12 \mathrm{Gyr}$. The estimate of the time for a ripple to appear at $2.2 \mathrm{kpc}$ from the galaxy center is $\sim 200 \mathrm{Myr}$, as indicated in our simulations giving $\Delta R=1.32 \mathrm{kpc}$.

Figure 1 illustrates, in its top panels, the time evolution of the disk displayed face-on for approximately 2 Gyr. Next, we computed the the first moment of the $z$ component of stars: $z_{0}=\Sigma z_{i} / N$, with $N$ being the number of stars at that radius, and from this the characteristic scale height of the disk, $z_{\text {disk }}$, during its evolution. To do this, it is convenient to parametrize the function $\zeta(z)$ of Equration (1) such that $\int d z \zeta(z)=1$ (Binney \& Tremaine 2008). With this choice, the quantity $\Sigma(R) \zeta(z) d z$ is the surface density of the layer of stars with thickness $d z$ which lies at a distance $z$ from the midplane. Figure 1 shows a time sequence of the face-on disk with a map of the the average vertical component, $z_{0}$ and the characteristic disk scale height, $z_{\text {disk }}$ (middle and bottom panels, respectively).

\subsection{Vertical and Horizontal Stellar Motions Coupled}

As the disk wobbles and waves of enhanced density move and form patterns similar to rings, we note that the underdense regions coincide with a local increase of the characteristic scale height, $z_{\text {disk }}$, at that specific location in the disk plane, as illustrated in Figure 1. 

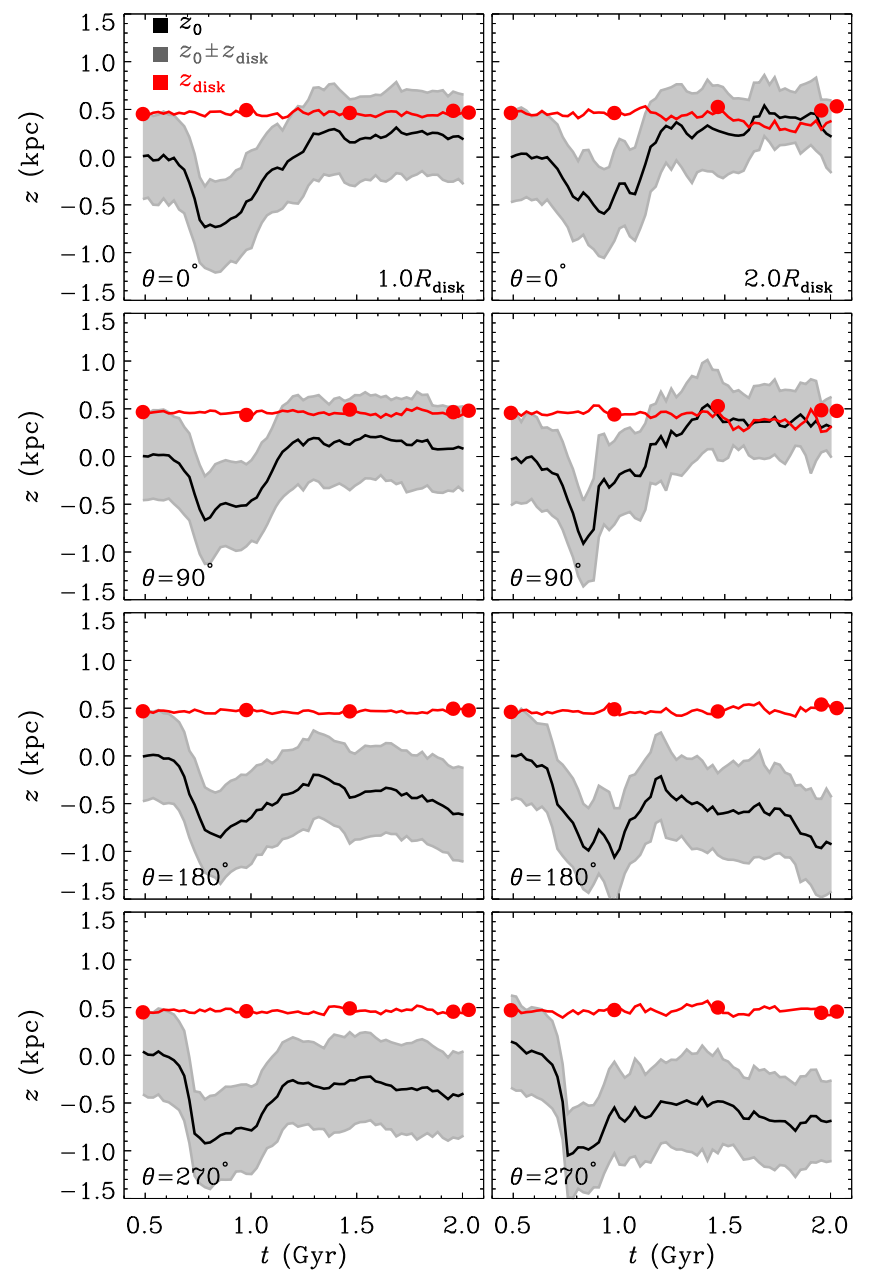

Figure 3. Left panels: time sequence of the average vertical component (solid black line) of stars located at $R_{d}=4 \mathrm{kpc}$, for different azimuthal angles: $\theta=0 ; 90 ; 180 ; 270$ for the stellar disk perturbed by one massive satellite with $M=2 \cdot 10^{10} M_{\odot}$ (described as first numerical experiment). The gray area is the $1 \sigma$ vertical displacements around the average value. The disk thickness is also displayed as a function of time (solid red line). Right panels: the same sequence as above is displayed for stars located at $2 R_{d}=8 \mathrm{kpc}$.

In galactic disks, stars are always assumed to be on nearly circular orbits with their motion usually well-described by the epicyclic approximation, and where the in-plane and vertical displacements of a star are decoupled. Stars near the disk midplane oscillate vertically with a frequency of $\nu_{z}^{2}=\frac{\partial^{2} \Phi}{\partial R^{2}}$, where $\Phi$ is the gravitational potential. For a Hernquist density profile, the potential is

$$
\Phi(r)=-\frac{G M}{r+a},
$$

where $a$ is the halo scale length, $G$ is the gravitational constant, and $M$ is the total halo mass. Then, the vertical oscillation frequency is

$$
\begin{aligned}
\nu_{z}^{2}= & \frac{\partial^{2} \Phi}{\partial z^{2}}=G M \\
& \cdot \frac{a\left(x^{2}+y^{2}\right)+\left(x^{2}+y^{2}-2 z^{2}\right) \sqrt{x^{2}+y^{2}+z^{2}}}{\left(x^{2}+y^{2}+z^{2}\right)^{3 / 2}\left(\sqrt{x^{2}+y^{2}+z^{2}}+a\right)^{3}}
\end{aligned}
$$

Note that the vertical frequency is usually greater than the epicyclic frequency. This means that the stars oscillate in their radial motion more slowly than in their vertical oscillations. This condition is particularly valid for a star located in a region of enhanced density, like in a density wave where, due to the stronger gravitational field, it will be forced to oscillate fast relative to the disk midplane. However, a star located in an underdense region feels a weaker gravitational restoring force, and has its vertical frequency reduced to roughly match the circular frequency, specifically, $\Omega \sim \nu_{z}$. Thus, for stars preferentially located in underdense regions, the horizontal motion will be coupled in frequency to the vertical motion. The overall effect of this unexpected coupling is to produce local enhancements of the characteristic disk scale height as illustrated in Figure 1.

\subsection{Stellar Vertical Oscillations and Heating}

Ideally, one would like to use the impulse approximation to study the energy deposited into the disk by a satellite passage. For a satellite of mass $M_{\text {sat }}$ moving at relative speed $V_{0}$ and impact parameter $b$, the mean change in the vertical kinetic energy of a disk star during a single satellite passage with random orientation is (Spitzer \& Hart 1971; Sellwood et al. 1998; D’Onghia et al. 2010b)

$$
\Delta E_{z} \propto z_{\text {disk }}^{2}\left(\frac{G M_{\text {sat }}}{b^{3} \nu_{z}}\right) \beta^{2}
$$

with the parameter $\beta=2 \nu_{z} b / V_{0}$ being the characteristic passage time of the satellite divided by the stellar orbital period. For a high-velocity satellite, the net vertical energy transferred is low since it is nearly the same as for a free particle, and thus varies as $V_{0}^{2}$. At low velocities, however, the energy transfer is exponentially small. However, a selfgravitating disk supports collective modes, which include vertical waves and horizontal density waves that are not included in the impulse approximation. When these collective effects are properly accounted for (e.g., using numerical simulations of sufficient resolution of self-gravitating disks with low noise), the deposition of vertical energy into the stars in the disk by the passage of a satellite has been demonstrated to be greatly reduced relative to the impulse approximation (Sellwood et al. 1998; Hopkins et al. 2008; Moetazedian \& Just 2015).

It has been argued that although a satellite passage does not heat a thin disk, there is substantial heating at late stages, through the process of exciting disk-bending waves. Once excited, these waves eventually dampen efficiently at waveparticle resonances, thereby heating the outer part of the disk (Sellwood et al. 1998). It should be noted that this assumes that the in-plain motion is decoupled from the vertical motion.

The outcome of our experiment with only the most massive satellite with $M=2 \times 10^{10} M_{\odot}$ hitting the disk is illustrated in Figure 2, which displays a map of the radial, tangential, and vertical velocity dispersion of stars at the time before the satellite impacts the disk, and at later times. It shows that there is some increase of random motions in the in-plane motion of stars or the vertical heating of the disk after more than $2.5 \mathrm{Gyr}$.

To demonstrate our findings, we evaluate the first moment of the $z$ component of stars, $z_{0}=\Sigma z_{i} / N$, and the second moment, $z_{\text {disk }}=\sqrt{\Sigma z_{i}^{2} / N}$, with $N$ being the number of stars at that radius. Figure 3 displays the evolution in time of the first 

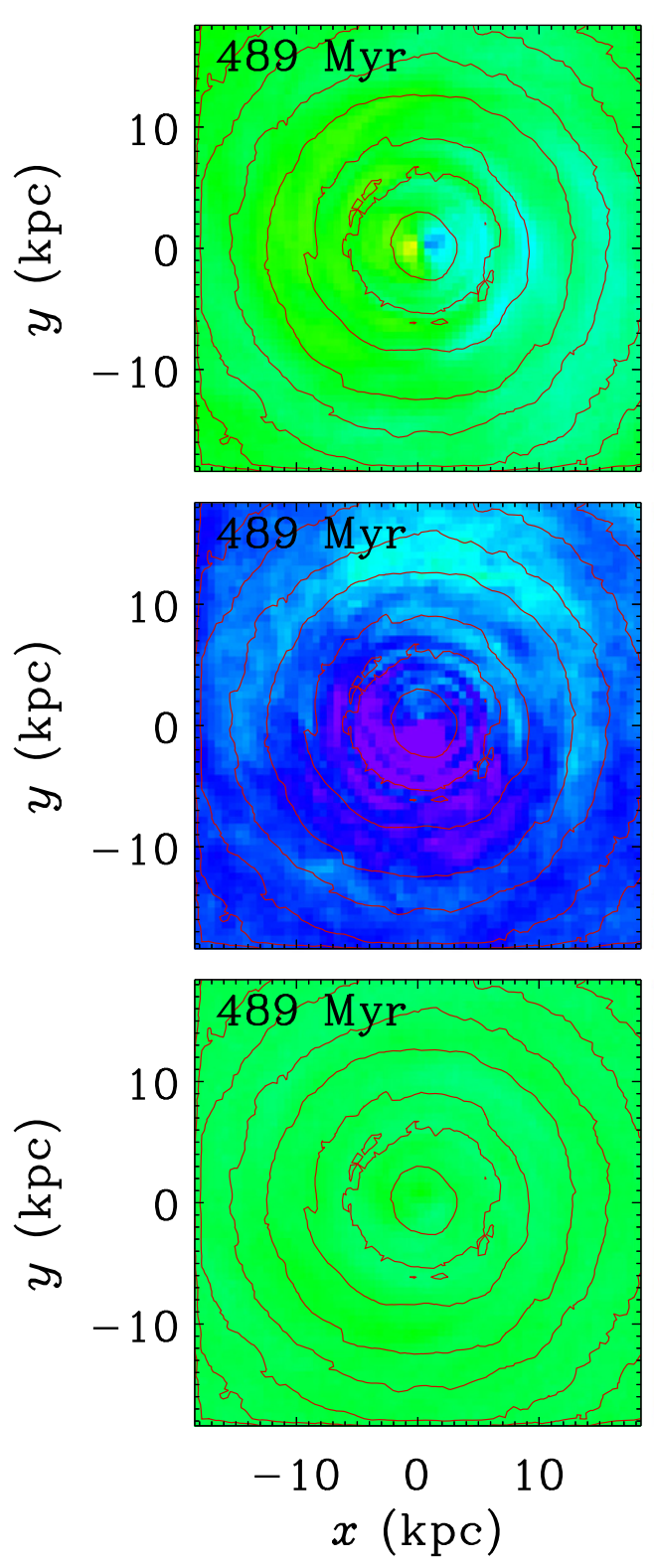

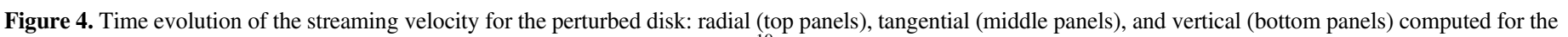

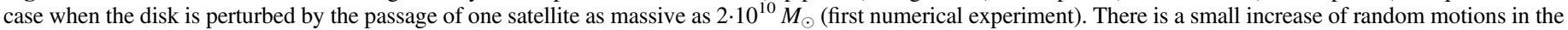
in-plane motion of stars or vertical heating the disk after more than $2.5 \mathrm{Gyr}$.

moment of the vertical component of the stars, $z_{0}$, (solid black line) evaluated at each panel for different azimuthal angles $(\theta=0,90,180)$ and at different radii, $R=4 \mathrm{kpc}$ (left panels) and $R=8 \mathrm{kpc}$ (right panels), respectively.

It seems clear from our results that the satellite impact induces the disk to wobble, with local enhancements of the characteristic scale length of the disk in underdense regions but with some increase of the disk thickness with time as illustrated by the evolution of $z_{\text {disk }}$ in Figure 3 (solid red line).

Could features such as the vertical displacements identified in our simulations be a manifestation of bending waves or breathing modes? Our N-body simulations involve self-gravitating disks; thus, they support collective modes. To address this question, we focus on this run when only the most massive substructure hits the disk, to isolate the phenomenon from the cumulative effects of many encounters with smaller substructures. If a massive satellite impacting the disk excites the formation of bending waves, there are good arguments to believe that these waves are characterized by long wavelengths that dampen and produce heating in the outer parts of the disk (Weinberg 1991).

Instead, we note vertical displacements in all of the underdense regions which we attribute to the effect of reduced restoring forces locally in the disk. This explanation is supported by the fact that these enhancements in vertical motions involve a modest number of stars and modest associated heating, as shown in Figures 2-3.

An interesting feature of our disk when hit by one massive satellite is that, at a distance corresponding to the solar neighborhood, the vertical displacement of stars in their motion perpendicular to the plane can extend up to $1 \mathrm{kpc}$ from the midplane with asymmetries in the number density of stars above and below the disk. These features are confirmed by Figure 4 where maps of the velocity streaming motions-radial, tangential, and vertical-for stars in the disk are given. Note that 


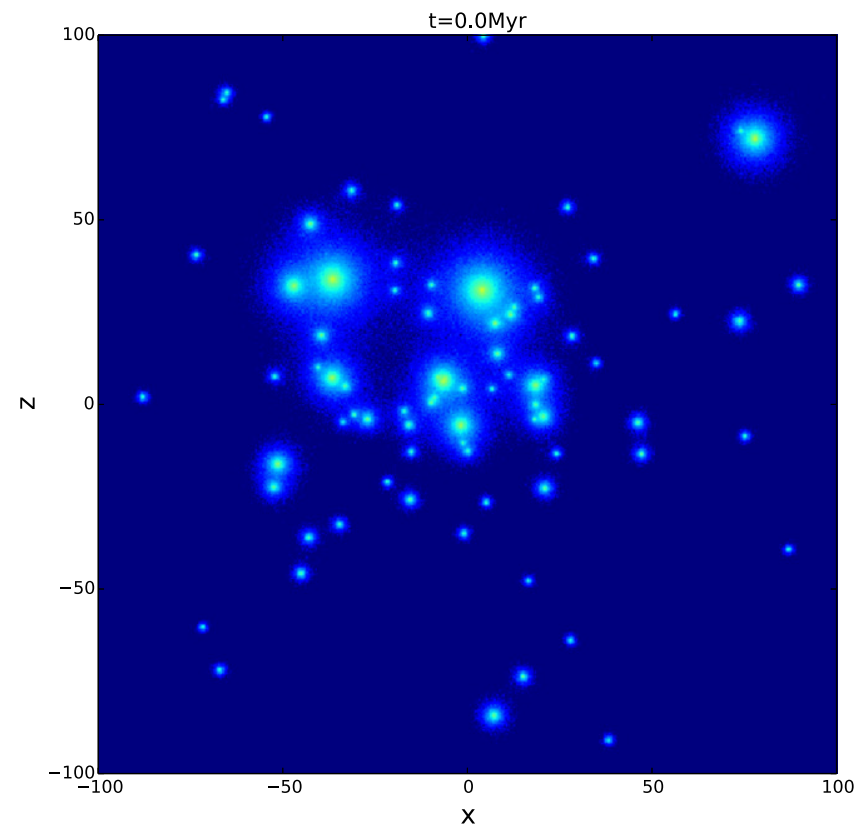

Figure 5. Spatial distribution of $\approx 100$ live dark subhalos with structural properties inferred from cosmological simulations from $2 \times 10^{8} M_{\odot}$ to $2 \times 10^{10} M_{\odot}$ (third numerical experiment).

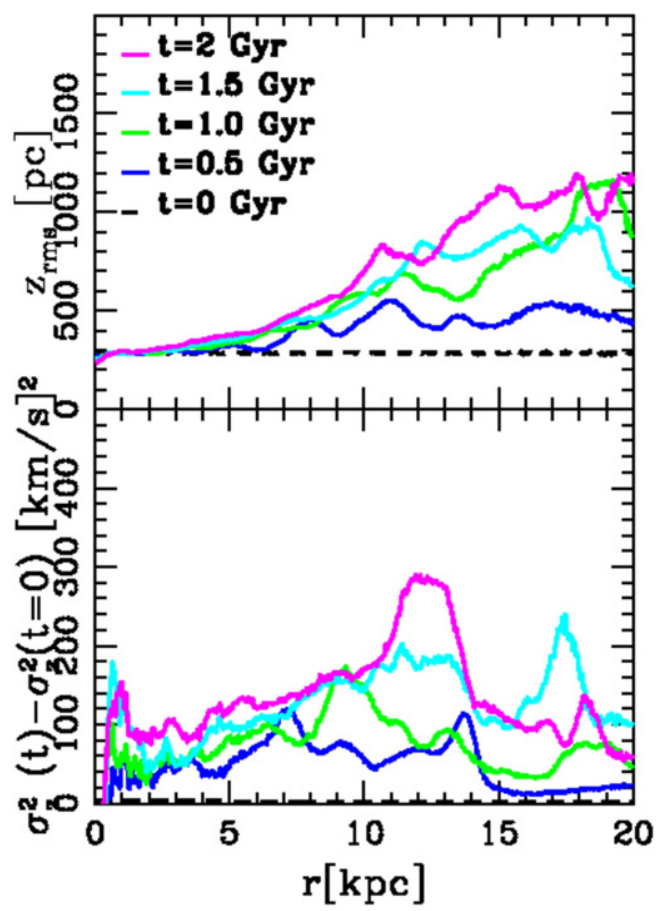

Figure 6. Top panel: time sequence of the first moment of the $\mathrm{z}$ component of stars as a function of radius for the experiment with several satellites hitting the disk (third numerical experiment). Bottom panel: the change of the vertical random energy from the initial time.

Figure 4 shows that the vertical streaming motions can be as large as $15-20 \mathrm{~km}^{-1}$ and are related to the wobbles in the disk.

\subsection{Dynamical Response of the Disk to Many Satellites}

We performed two additional N-body experiments in which a live disk of $10^{8}$ star particles was embedded in a rigid potential with the introduction of substructures.
In one experiment, we added to the live disk, which is embedded in a static potential for the halo, 1000 substructures with a mass of $10^{7} M_{\odot}$ hitting the disk from random orbits (labeled as second experiment), and found that the disk did not develop prominent features in this situation, as expected from the analysis of Hopkins et al. (2008). In the other experiment, the same galaxy model was evolved but with the introduction of $\approx 100$ live dark satellites isotropically distributed (named the third experiment).

Figure 5 illustrates the spatial distribution of the $\approx 100$ live dark subhalos that are placed on orbits with eccentricities ranging from 0.3 to 0.9 , around the stellar disk, which is run in isolation for $500 \mathrm{Myr}$ to achieve equilibrium prior to interacting with the substructures. The disk is not displayed in the image.

On the contrary, in the simulation where the disk is bombarded by $\approx 100$ satellites, there is considerable vertical random energy in the disk. Figure 6 displays the time evolution of the first moment of the $z$ component of stars as a function of radius, $z_{0}$, and $\sigma_{z}^{2}(t)-\sigma_{z}^{2}(t=0)$, that is, the change of the vertical heating from the initial time for the experiment with several satellites orbiting around the disk. This result confirms the previous findings that disk heating is caused by a few massive satellites passing through the disk (Hopkins et al. 2008; Moetazedian \& Just 2015), although their velocity also plays a role. We note that in this case, the vertical heating is not sudden but gradually increases as time progresses. Although after $2 \mathrm{Gyr}$ there is considerable heating produced in the solar neighborhood, the heating and flaring of stars are very pronounced in the outer part of the disk, which is in agreement with the results of the disk run AQ-F2 presented in Moetazedian \& Just (2015).

In this case, it is possible that several impacts of massive satellites with different relative velocities with respect to the disk represent perturbations with a spectrum of higher frequencies in the range that excites the formation of long-bending waves that, once damped, can heat the disk and generate flares.

\section{SUMMARY AND CONCLUDING REMARKS}

The goal of our study has been to gain physical insight into the gravitational response of a disk to perturbations induced by satellites passing through it, as predicted by cosmological simulations. We have chosen to focus mainly on the impact of one satellite as massive as the Sagittarius dwarf galaxy in order to isolate the phenomenon from the cumulative effects of many encounters with smaller substructures.

We show that a not-too-fast satellite as massive as Sagittarius passing through the disk can induce the disk to wobble and form patterns similar to in-plane rings, which is in agreement with previous results. However, our findings indicate that the underdense regions between rings overlap with local enhancements in disk thickness. This coincidence is due to stars located in underdense regions that have coupled horizontal and vertical motions. Those stars display vertical displacements that can extend up to $1 \mathrm{kpc}$ with non-zero vertical streaming motions as large as $15-20 \mathrm{~km} \mathrm{~s}^{-1}$, which is consistent with recent observations in the solar neighborhood. This phenomenon appears like local flaring. However, the dark halo is spherical in these numerical experiments. This assumption might artificially slow down the precession rates of the oscillations, delaying the disk heating.

In the simulation with $\approx 100$ satellites injected around the stellar disk with mass and properties expected in cosmological simulations, our results indicated that disk flaring is produced 
in the outer parts of the disk with significant vertical heating experienced by the stellar disk within $2.5 \mathrm{Gyr}$.

Given the fact that Sagittarius likely passed through the disk and that the Sun is located in an underdense region, our picture naturally predicts that in the solar neighborhood the mass density of the disk estimated by $V_{z}$ dispersion measurements of stars will be over-estimated compared to other regions of the disk. This over-estimate, known as the problem of the Oort limit of the stellar disk, once reconciled, might indicate a lower mass-to-light ratio in the disk, suggesting that there is almost no need for dark matter at the position of the Sun.

This work is funded by NSF grant no. AST-1211258 and ATP-NASA grant no. NNX14AP53G. E.D. gratefully acknowledges the support of the Alfred P. Sloan Foundation and the hospitality of the Aspen Center for Physics, funded by NSF grant no. PHY-1066293. P.M. acknowledges support by the NSF through grant AST-1229745 and NASA through grant NNX12AF87G. L.H. acknowledges support from NASA grant NNX12AC67G and NSF grant AST-1312095.

\section{REFERENCES}

Binney, J., \& Tremaine, S. 2008, Galactic Dynamics (2nd ed; Princeton, NJ: Princeton Univ. Press)

Bird, J. C., Kazantzidis, S., \& Weinberg, D. H. 2012, MNRAS, 420, 913

Carlin, J. L., DeLaunay, J., Newberg, H. J., et al. 2013, ApJL, 777, L5

Chakrabarti, S., \& Blitz, L. 2009, MNRAS, 399, L118

de la Vega, A., Quillen, A. C., Carlin, J. L., Chakrabarti, S., \& D’Onghia, E. 2015, MNRAS, 454, 933

Debattista, V. P. 2014, MNRAS, 443, L1

Diemand, J., Kuhlen, M., Madau, P., et al. 2008, Natur, 454, 735

D’Onghia, E. 2015, ApJL, 808, L8

D'Onghia, E., Mapelli, M., \& Moore, B. 2008, MNRAS, 389, 1275

D’Onghia, E., Springel, V., Hernquist, L., \& Keres, D. 2010a, ApJ, 709, 1138
D’Onghia, E., Vogelsberger, M., Faucher-Giguere, C.-A., \& Hernquist, L. 2010b, ApJ, 725, 353

D’Onghia, E., Vogelsberger, M., \& Hernquist, L. 2013, ApJ, 766, 34

Gómez, F. A., Minchev, I., O'Shea, B. W., et al. 2012, MNRAS, 423, 3727

Gómez, F. A., Minchev, I., O'Shea, B. W., et al. 2013, MNRAS, 429, 159

Gómez, F. A., White, S. D. M., Marinacci, F., et al. 2016, MNRAS, 456, 2779

Hernquist, L. 1990, ApJ, 356, 359

Hernquist, L. 1993, ApJS, 86, 389

Hernquist, L., \& Weil, M. L. 1993, MNRAS, 261, 804

Hopkins, P. F., Cox, T. J., Younger, J. D., \& Hernquist, L. 2009, ApJ, 691, 1168

Hopkins, P. F., Hernquist, L., Cox, T. J., Younger, J. D., \& Besla, G. 2008, ApJ, 688, 757

Kazantzidis, S., Zentner, A. R., Kravtsov, A. V., Bullock, J. S., \& Debattista, V. P. 2009, ApJ, 700, 1896

Lynds, R., \& Toomre, A. 1976, ApJ, 209, 382

Mapelli, M., \& Mayer, L. 2012, MNRAS, 420, 1158

Moetazedian, R., \& Just, A. 2015, arXiv:1508.03580

Monari, G., Famaey, B., \& Siebert, A. 2015, MNRAS, 452, 747

Monari, G., Helmi, A., Antoja, T., \& Steinmetz, M. 2014, A\&A, 569, A69

Purcell, C. W., Bullock, J. S., Tollerud, E. J., Rocha, M., \& Chakrabarti, S. 2011, Natur, 477, 301

Quinn, P. J., Hernquist, L., \& Fullagar, D. P. 1993, ApJ, 403, 74

Sellwood, J. A. 2013, ApJL, 769, L24

Sellwood, J. A., Nelson, R. W., \& Tremaine, S. 1998, ApJ, 506, 590

Spitzer, L., Jr., \& Hart, M. H. 1971, ApJ, 164, 399

Springel, V., Di Matteo, T., \& Hernquist, L. 2005, MNRAS, 361, 776

Springel, V., Wang, J., Vogelsberger, M., et al. 2008, MNRAS, 391, 1685

Struck, C., Dobbs, C. L., \& Hwang, J.-S. 2011, MNRAS, 414, 2498

Toth, G., \& Ostriker, J. P. 1992, ApJ, 389, 5

Vera-Ciro, C., D’Onghia, E., Navarro, J., \& Abadi, M. 2014, ApJ, 794, 173

Walker, I. R., Mihos, J. C., \& Hernquist, L. 1996, ApJ, 460, 121

Weinberg, M. D. 1991, ApJ, 373, 391

Widrow, L. M., Barber, J., Chequers, M. H., \& Cheng, E. 2014, MNRAS, 440, 1971

Widrow, L. M., Gardner, S., Yanny, B., Dodelson, S., \& Chen, H.-Y. 2012, ApJL, 750, L41

Williams, M. E. K., Steinmetz, M., Binney, J., et al. 2013, MNRAS, 436, 101 Yanny, B., \& Gardner, S. 2013, ApJ, 777, 91

Younger, J. D., Besla, G., Cox, T. J., et al. 2008, ApJL, 676, L21 